\title{
Research on Industrial Waste Recovery Network Optimization: Opportunities Brought by Artificial Intelligence
}

\author{
Bin Liao $(\mathbb{D})$ and Ting Wang $(\mathbb{D}$ \\ School of Management, University of Guizhou, Guiyang, China \\ Correspondence should be addressed to Ting Wang; wting74@163.com
}

Received 3 November 2019; Revised 26 February 2020; Accepted 23 March 2020; Published 25 April 2020

Guest Editor: Davide Castellano

Copyright ( 92020 Bin Liao and Ting Wang. This is an open access article distributed under the Creative Commons Attribution License, which permits unrestricted use, distribution, and reproduction in any medium, provided the original work is properly cited.

\begin{abstract}
With the acceleration of industrialization and urbanization in China, a large amount of waste in industrial parks has become the main cause of regional environmental pollution. In order to solve this problem, this paper relied on artificial intelligence's prediction technology and image recognition technology to intelligently upgrade the traditional industrial waste planning management system and designed a waste intelligent classification center with intelligent prediction and intelligent classification capabilities. So, as to realize this new intelligent classification center and explain its value, this paper explains the key implementation technology of this intelligent classification center and validates it by constructing a multitarget location model that considers both economic and environmental benefits.
\end{abstract}

\section{Introduction}

With the rapid development of industry and economy, the problem of environmental pollution is becoming more and more serious. In the face of the "three great mountains" of competition proliferation, resource depletion, and environmental degradation, governments have begun to attach great importance to the coordinated balance between economic development and environmental protection issues. As of 2017, China produces about 3.3 billion tons of industrial waste each year, with a total stockpiled over 60 billion tons over the years. Industrial waste that has not been disposed of in a timely manner has not only caused a huge waste of land resources but also caused regional ecosystem disturbances [1-3]. How to safely, economically, environmentally, and efficiently treat these wastes has become one of the important research topics for the development of a sustainable economy in China.

The social demand for recovering and reusing discarded products has stimulated a research boom in product-recycling networks [4]. After years of accumulation, the research on the waste product-recycling network has achieved results, but it mainly focuses on the recovery and reuse of mobile phones, automobiles, washing machines, and other electronic products. Few studies have discussed the issue of industrial waste recycling [5-9]. In the existing research on industrial waste management, most scholars treat industrial waste and domestic waste as urban waste for discussion. Huang and Li discussed the problems of Wuhan municipal waste recycling, treatment, and resource reuse. Finally, they proposed the scientific classification of municipal waste and diversified garbage collection, charging, and treatment management methods to promote Wuhan's waste management related development of the industry [10]; Li et al. proposed an urban waste recycling system based on radio frequency wireless network, including system function description, hardware design, and software design. The system uses sensor technology and radio frequency wireless network technology to realize the information collection of urban garbage bins and the positioning of urban garbage collection systems. This research has realized the dynamic tracking and monitoring of garbage from the source to the disposal terminal and has made some contributions to the intelligent development of garbage recycling management [11]; Wang et al. established a game relationship model for residents, receiving and transporting enterprises, and waste 
processing enterprises under different classification ratios based on the Stackelberg game theory. The proportion of the responsibility of the waste management of the subject and the simulation analysis of the model show that the waste classification capacity of industrial waste service enterprises is the main factor affecting the interests of the subjects in the recycling network [12].

Importantly, as the process of industrialization and urbanization continues to accelerate, the large concentration of industries and populations has saturated the load of the previous urban waste transportation network, which has led to a sudden decline in the efficiency of the traditional industrial waste. As a result, severe waste accumulation and environmental pollution have occurred in many regions of China. Therefore, the separation of traditional waste recycling systems and the design of a recycling management network dedicated to the treatment of industrial waste to improve the efficiency of waste treatment are becoming research hotspots in the field of industrial waste management and reuse.

In the research of industrial waste recycling management network, scholars mostly discuss the industrial waste network planning and design based on the perspectives of logistics cost, service level, and equipment utilization efficiency [13-15]. Wang et al. constructed a two-objective optimization model when designing the logistics network to analyze the impact of different input costs on the environment in the design of the supply chain while considering the two goals of cost and environmental protection level to analyze the project's return on investment. The rate has a positive enlightening effect on the future green management of the supply chain [16]. Since then, Elhedhli and Ryan further explore the relationship between distance and carbon emissions [17]; Rui et al. considered a logistics network planning model with a hard time window and analyzed the impact of different time windows on the economy and the environment [18]; Zhang and Li-rong others have considered the uncertainty of the quality of the recycled products in the market, set up a multiobjective stochastic programming model with the goal of minimizing logistics network costs and environmental pollution, and solved the stochastic programming model with scenario analysis [19].

This thesis considers the intelligent upgrading of industrial waste recycling management network in an industrial park with multiple factories. First, the artificial intelligence prediction technology and image recognition technology are used to intelligently upgrade the traditional industrial waste planning management system. An intelligent classification center for industrial waste with intelligent prediction and intelligent classification capabilities is designed, and its key implementation technology is discussed. Finally, the value and significance of the intelligent classification center designed in this paper are illustrated by constructing a multiobjective site selection model considering both economic and environmental benefits.

The rest of this article is organized as follows. The next section puts forward the key technologies for the realization of the industrial waste intelligent classification center system architecture, including the industrial waste production and component prediction models based on BP learning algorithms and deep learning-based waste identification and detection implementation technologies. In the fourth section, the site selection model, implementation algorithm, and numerical simulation of the industrial waste intelligent recycling center considering economic and environmental benefits are designed. The last section discusses and summarizes the thesis work.

\section{Design of Artificial Intelligent Waste Sorting Center}

2.1. System Framework of Artificial Intelligence Recycling Classification Center. The optimization of an industrial waste recycling network system is essentially a multilevel, multiobjective management decision problem. To upgrade the traditional model, it is necessary to redesign a decision model with corresponding functions. In this research, artificial intelligence technology is integrated into the traditional waste logistics recycling system. The system framework of the artificial intelligence recycling classification center that integrates the two core technologies of waste production prediction technology and image recognition is designed, as well as its functional decision model. It can be seen from Figure 1 that this intelligent recycling classification center has basic decisionmaking functions for industrial waste production forecasting, component forecasting, image recognition, and intelligent location selection. During the operation of the classification center, the quantity and category prediction of the waste to be processed is first based on the preset related parameters. On the one hand, these prediction results can be used as training sets to train and detect the image recognition model. On the other hand, it can be combined with processing power as a parameter source for the location model of the intelligent classification center to verify the economic and environmental benefits of such intelligent classification centers replacing traditional artificial classification centers. It can be seen that the data flow is progressively advanced between different models, forming an organic whole with complex relationships.

\subsection{Key Technologies Implemented by Artificial Intelligence} Recycling Classification Center. As can be seen from the above, the basis for realizing the artificial intelligence classification center designed in this paper is the design of industrial waste prediction technology and image recognition technology. In this section, we focus on the design ideas and algorithms of these two technologies.

2.2.1. Prediction Model of Industrial Waste Products and Components Based on BP Learning Algorithm. The neural network is a kind of multilayer feedforward neural network trained according to the error backpropagation algorithm. It is the most widely used neural network. The artificial neural network does not need to determine the mathematical equation of the mapping relationship between input and output in advance. It only learns certain rules through its own training and obtains the result closest to the expected output value when given the input value. With the 


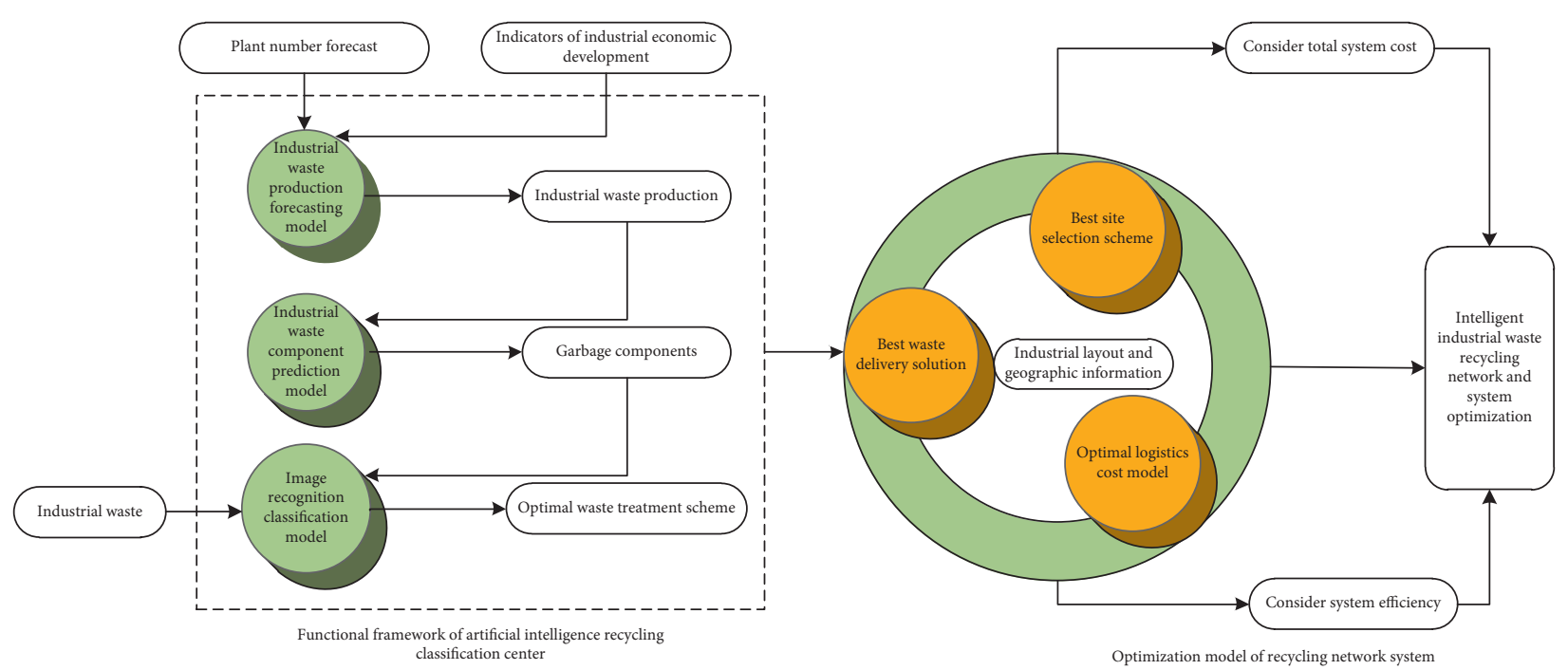

FIGURE 1: System framework of artificial intelligence recycling classification center and its functional decision-making model.

advantages of high prediction accuracy and robust results, this method is widely used in intelligent prediction, machine learning, and other fields [20]. This paper designs the BP network prediction model of industrial waste products and composition, as shown in Figure 2.

The core of an artificial neural network to realize its function is the algorithm. BP neural network is a multilayer feedforward network trained by error backpropagation. The BP learning algorithm is an error correction method based on this principle. The learning process of the algorithm consists of forwarding and backpropagation (partial composition). When an input mode of a network is given, it is passed from the input layer unit to the hidden layer unit, processed by the hidden layer unit, and then sent to the output layer unit, and an output mode is generated by the output layer unit. Because this is a layer-by-layer effect in the process, each layer of neurons can only affect the state of the next layer of neurons, which is called forward propagation; if the output response is in error with the expected output mode and does not meet the requirements, then the error is transferred to the backpropagation and then transmitted. The value is transmitted layer by layer along the connection path, and the connection weight of each layer is modified. For a set of samples, learning is performed by using different training modes, and the model is continuously repeated in the forward and backward propagation processes. Only when each training mode meets the requirements, the BP network training is completed. Network learning is a process of minimizing the objective function to complete the inputto-output mapping. Generally, the objective function is defined as the sum of the squared error of the output layer unit's desired output and the actual output on all input modes. The prediction algorithm of industrial waste production and composition in this paper is as follows:

Step 1: suppose the input layer has nneurons, the input vector is $X\left(x_{1}, x_{2}, \ldots, x_{n}\right)$; the hidden layer has $m$ neurons, the hidden layer vector is $H\left(h_{1}, h_{2}, \ldots, h_{m}\right)$; the output layer has $k$ neurons, the output vector is
$Y\left(y_{1}, y_{2}, \ldots, y\right)_{k}$. The weight between the input layer and the output layer is and the threshold is ; the weight between the hidden layer and the output layer is and the threshold is.

Step 2: assign initial values to all connection weights and node thresholds

Step 3: do the following calculations for each input sample:

(a) Forward calculation:

$$
\left\{y_{k}=\sum_{j=0}^{m} \omega_{j k \cdot h_{j}}, h_{j}=\sum_{j=0}^{n} \omega_{i j \cdot x_{i}} .\right.
$$

Let the transfer function be a sigmoid function, that is, $f(x)=(1 / 1+\exp (-a x))(a>0)$.

For the $P_{i}$ sample in the training set, the network input vector is $X\left(x_{1}, x_{2}, \ldots, x_{n}\right)$, the actual output is $Y\left(y_{1}, y_{2}, \ldots, y_{k}\right)$, the expected output is $T\left(t_{1}, t_{2}, \ldots, t_{n}\right)$, and the error function is defined as follows:

$$
E=\frac{1}{2} \sum_{k=1}^{i}\left(t_{k}-y_{k}\right)^{2}
$$

Let the total number of samples is $N$, then the mean of the squared error is

$$
E_{A V}=\frac{1}{N} \sum_{N=1}^{N} E
$$

$E_{A V}$ is the mean of the squared error. The purpose of the algorithm learning is to minimize $E_{A V}$.

(b) Inverse calculation

When the algorithm fails to meet the target expectations, it will calculate backward. The first is to calculate the local gradient $\delta_{k}$. The first is to calculate 


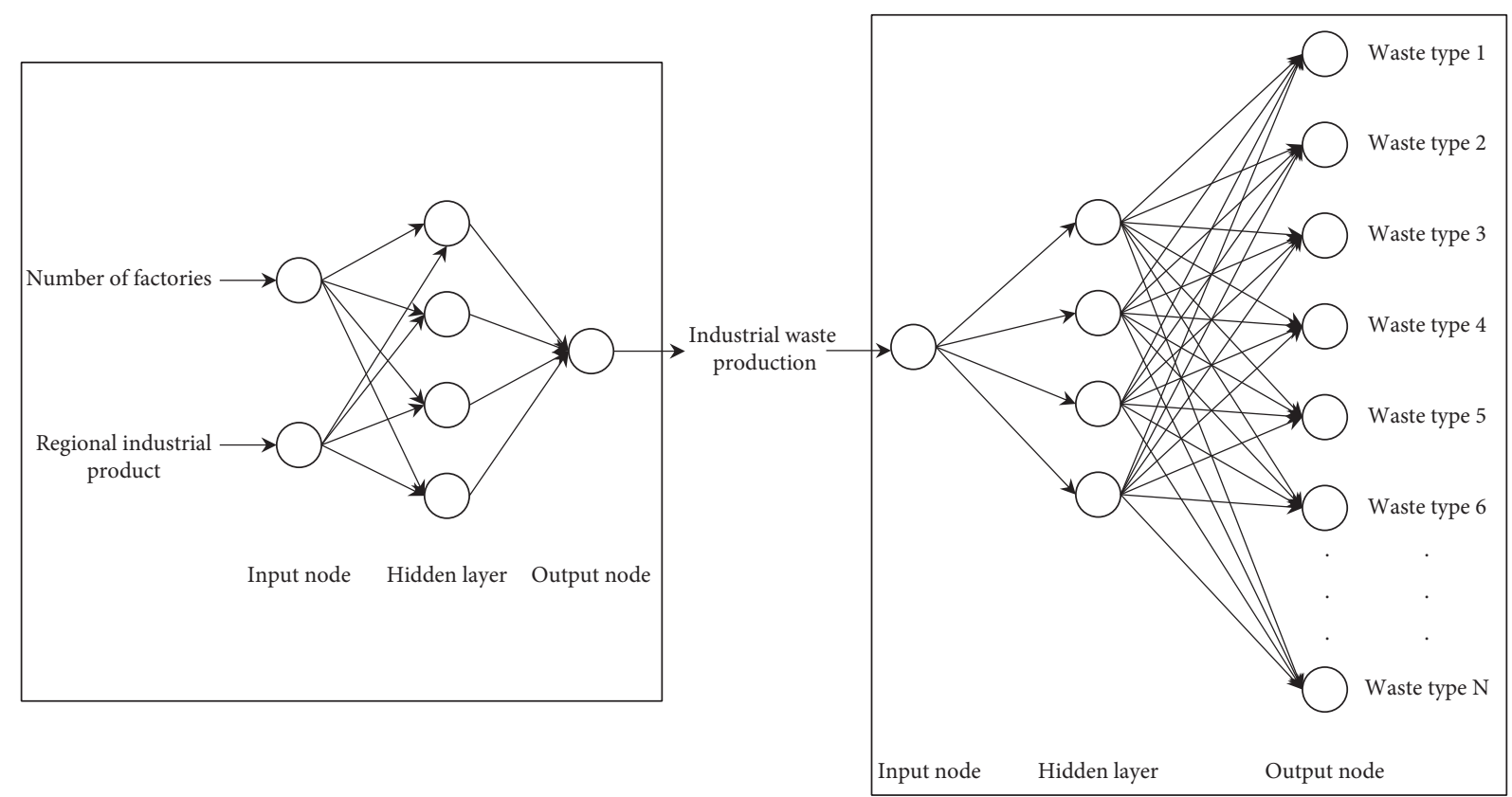

FIGURE 2: BP network prediction model for industrial waste products and components.

the local gradient. It is worth noting that for the output and hidden layers, the algorithms of the local gradient are different, as shown in formulas (4) and (5):

$$
\begin{aligned}
& \text { Output layer } \delta_{k}=\left(t_{k}-y_{k}\right) f\left(x_{k}\right), \\
& \text { Hidden layer } \delta_{k}=\frac{\delta E}{\delta y_{k}} f\left(x_{k}\right) .
\end{aligned}
$$

\section{(c) Correction weight:}

Using the minimum value of the gradient descent method, the updated amount $\Delta \omega_{i j}$ of $\omega_{i j}$ can be expressed by the following formula:

$$
\Delta \omega_{i j}=-\eta \frac{\partial E}{\partial \omega_{i j}} .
$$

$\eta$ represents the learning rate of the neural network, and its value is greater than zero.

Step3: enter a new sample until $E_{A V}$ meets the predetermined requirements. Eventually, the model can output the annual industrial waste output and composition of the region. These data will be used as a training sample for image data and site selection for training to implement waste identification and monitoring technology data source for the model.

\subsubsection{Realization Technology of Waste Recognition and} Detection Based on Deep Learning. Deep learning is an emerging research field of machine learning. Its research content is to automatically extract multilayer features from the data and represent them. In the process, a series of nonlinear transformations can be used to extract features from the original data and finally realize machine vision and intelligent operation such as machine sensing. This research uses the idea of deep learning to design the target detection technology of industrial waste images and uses the type, position, size, and confidence of the target object as the determination parameters to achieve intelligent detection and recognition of the predetermined target object. The basic flow of the target detection technology is shown in Figure 3.

The target detection algorithm is the key to achieve intelligent detection. Considering the complexity of industrial waste detection tasks in different environments, this study improves the Faster RCNN [21] target detection model, which combines the RPN network and Fast-RCNN Unified to identify the location and angle of industrial waste at the same time. Faster RCNN model, which is composed of two subnetworks: Region Proposal Generation (RPN) network and VGG-16 classification network as shown in Figure 4.

The RPN network adopts the design method of the fully convolutional network, in which the convolutional layer uses the VGG-16 model and shares convolutions with subsequent classification networks to reduce double counting. Figure 5 shows the structure of the RPN network. A small network is used to slide the feature image output by the last shared convolution layer to generate the region of interest. The input of this small network comes from the $n \times n$ size window input on the convolutional feature image, and then the features of each sliding window will be mapped to the lower dimensional feature vector. After the mapping, Re LU will perform nonlinear processing. The features, thus, obtained are used as input to two parallel fully connected layers-the bounding box return layer (REG) and the classification layer (CLS). The regression layer is responsible for readjusting the 


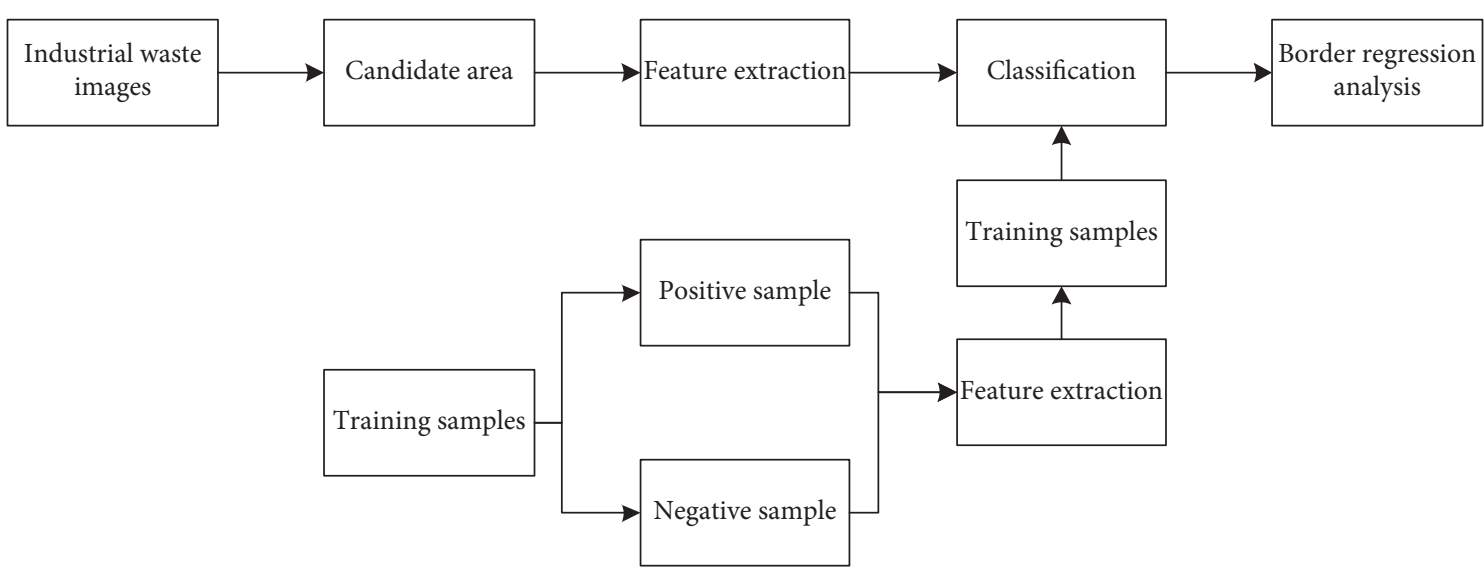

FigURE 3: Basic flowchart of industrial waste monitoring technology.

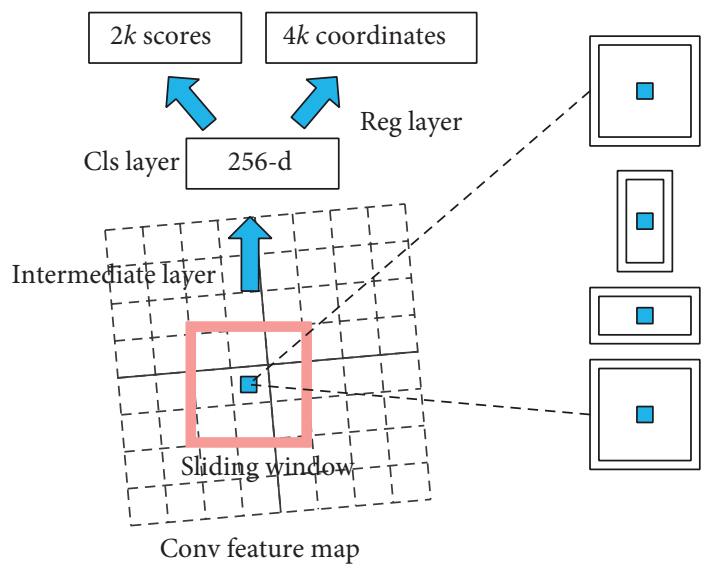

FigURE 4: RPN network structure.

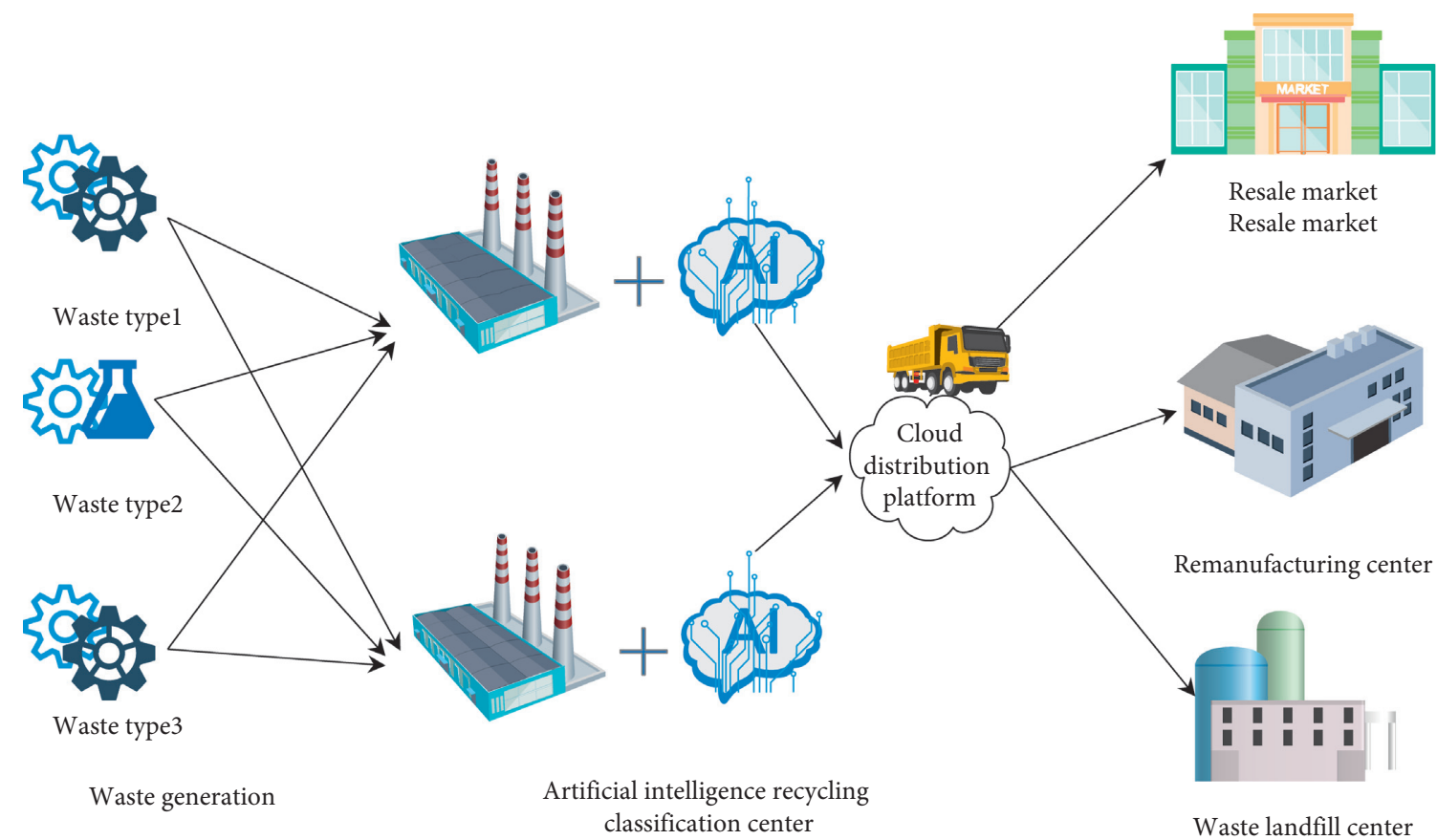

Figure 5: Industrial waste smart recycling model concept illustration. 
inaccurate area of interest, and the function of the classification layer is responsible for determining whether the area belongs to a candidate area. This processing method ensures that all features are associated with the REG and CLS layers. At the position of each sliding window, determine whether multiple regions of interest contain objects at the same time. The number of regions predicted simultaneously at each position is recorded as $k$. Thus, the bounding box regression layer outputs a total of $4 k$ coordinates for $k$ regions, and the classification layer outputs a total of $2 k$ scores to evaluate whether each region contains an object. In each sliding window position, 3 scales and 3 aspect ratios are used by default, so there are $k=9$ anchor points in total. So, for a featured image of size $w \times h$, there are " $w * h * k$ anchor points. After obtaining candidate regions of interest through the RPN network, the VGG-16 network pretrained on the ImageNet dataset is used for classification.

The VGG-16 network is a convolutional neural network structure proposed by the VGG group in 2014. The dataset is ImageNet. It uses 16 network layers with parameters, including 13 convolution kernels with a size of $3 \times 3$. It consists of 3 fully connected layers [22]. In addition, the same as Fast R-CNN, on the last convolutional layer, the spatial pyramid pooling (SPP) layer is also used to pool the feature layers to the same size. In the original RPN scheme, a decoupling scheme different from the traditional model is adopted, that is, the angle prediction layer is separately connected behind the copied FC layer of the VGG-16 network, so that it is not updated at the same time as the position of the industrial waste and the category layer. The scheme can further reduce the error of the bottle object angle prediction. As with Fast R-CNN, our training model uses a minimizing multitask loss function. The loss function of the RPN and VGG-16 networks is the same. The loss function consists of two terms in the following formula:

$$
L\left(\left\{P_{i}\right\},\left\{t_{i}\right\}\right)=\frac{1}{N_{\mathrm{cls}}} \sum_{i} L_{\mathrm{cls}}\left(p_{i}, p_{i}^{*}\right)+\lambda \frac{1}{N_{\mathrm{reg}}} \sum_{i} p_{i}^{*} L_{\mathrm{reg}}\left(t_{i}, t_{i}^{*}\right) .
$$

Among them, $i$ represents the pulling of anchor points in mini-batch and $P_{i}$ represents the probability that the $i$ anchor point is predicted to be an object. If the anchor point belongs to a positive sample, then $p_{i}^{*}$ is 1 , otherwise, it is $0 . t_{i}$ is a 5 -dimensional vector and belongs to the parameterized predicted object's bounding box coordinates, and $t_{i}^{*}$ is the $t_{i}$ label associated with the positive anchor. The classification loss function $L_{\mathrm{cls}}$ is calculated using a softmax loss strategy and the regression loss calculation is performed using a robust regression loss function (Smooth L1). When $p_{i}^{*}=1$, it means that the regression loss is only activated at the anchor point for processing positive samples. At this time, the outputs of the corresponding CLS and REG layers are $\left\{P_{i}\right\}$ and $\left\{t_{i}\right\}$.

Among them, for the graphs bounding box regression, we adopt the R-CNN parameterization method to unify the regression parameters:

$$
\begin{aligned}
& t_{x}=\left(\frac{\left(x-x_{a}\right)}{w_{a}}\right), \\
& t_{y}=\left(\frac{\left(y-y_{a}\right)}{h_{a}}\right), \\
& t_{x}^{*}=\left(\frac{\left(x^{*}-x_{a}\right)}{w_{a}}\right), \\
& t_{\mathrm{y}}^{*}=\left(\frac{\left(y^{*}-y_{a}\right)}{h_{a}}\right), \\
& t_{w}=\log \left(\frac{w}{w_{a}}\right), \\
& t_{h}=\log \left(\frac{h}{h_{a}}\right), \\
& t_{w}^{*}=\log \left(\frac{w^{*}}{w_{a}}\right), \\
& t_{h}^{*}=\log \left(\frac{h^{*}}{h_{a}}\right), \\
& t_{p}=\left(\frac{t}{2 \pi}\right), \\
& t_{p}^{*}=\left(\frac{t^{*}}{2 \pi}\right) .
\end{aligned}
$$

Among them, $x, y$, and $w$ represent the center coordinates of the frame, $h$ represents the length, $x_{a}$ represents the position of the anchor point, and $x^{*}$ and $y^{*}$ represent the position of the callout frame.

\section{Study on Site Selection of Intelligent Industrial Waste Recycling Center}

3.1. Problem Statement. The key technologies of the artificial intelligence recycling classification center have been explained above. It is not difficult to find that the artificial intelligence recycling classification center with image recognition technology and prediction technology can save a lot of labor costs. In order to verify the economic benefits of the designed artificial intelligence classification and processing center, from the perspective of system integrity, this paper designs a two-level goal of system planning research with system cost minimization and system efficiency maximization. The intelligent processing center will be integrated and sent to the waste data according to the operation process. At the same time, the optimal number and location of intelligent processing centers in the industrial waste processing network will be determined based on the geographical location of the various end-of-waste processing nodes. Finally, the economic benefits are judged by comparing the difference between the construction cost and the saved human resources.

Before this, it is necessary to briefly explain the operation mode of the entire waste system. This research integrates artificial intelligence technology into the traditional waste logistics recycling network and connects the various 
recycling nodes of industrial waste through the artificial intelligence classification center. The goal is to achieve the intelligence of the full recycling network, minimize costs, and maximize efficiency. The waste in the city is classified and processed (e.g., landfill, remanufacturing, and resale). Simplifying the problem can be summarized as the main point of industrial waste generation, artificial intelligence processing center, remanufacturing center, and landfill center, and for the small-cap market, the relationship is shown in Figure 5.

\subsection{Mathematical Formula}

3.2.1. Model Assumptions. In order to facilitate the analysis and description of the problem, this article makes some assumptions:

(1) All activities included in the recycling model proposed in this article are carried out in this model

(2) The system relies on existing secondary market, remanufacturing plant, no longer considers their construction costs, and only considers their operating costs

(3) The transportation costs of industrial waste are linearly related to the distance

(4) The waste output predicted by the trained neural network is equal to the real output

3.2.2. Model Parameter Setting and Description. The parameters involved in the model built in this paper mainly include node parameters, other parameters, general parameters, and decision variables, as shown in Table 1.

3.2.3. Objective Function and Constraints. The objective function considered in this paper is mainly the economic and environmental benefits of building an artificial intelligence classification center, so the following two objective functions are constructed:

$$
\begin{aligned}
\max F_{1}= & \left(\frac{\left(\sum_{k} Z_{m L}^{E R}+\sum_{k} Z_{k l}^{W R}\right)}{\sum_{j} Z_{j k}^{Q W}}\right) \\
\min F_{2}= & {\left[\sum_{k} b_{k}^{n}+\lambda\left(Z_{k l}^{W R}+Z_{m l}^{E T}\right)\right] } \\
& -\left[\sum_{k} p_{k}^{W}+\alpha \sum_{k} Z_{j k}^{Q W}+\beta \sum_{k} Z_{k m}^{W E}+\gamma\left(Z_{m n}^{W T}+Z_{m n}^{E T}\right)\right] \\
& -\left[\sum_{k} d Z_{j k}^{Q W} g+\sum_{k} d Z_{k m}^{W E} g+\left(\sum_{k} d Z_{m n}^{W T} g+\sum_{k} d Z_{m n}^{E T} g\right)\right. \\
& \left.+\left(\sum_{k} d Z_{k l}^{W R} g+\sum_{k} d Z_{m l}^{E R} g\right)\right]
\end{aligned}
$$

Among them, equation (9) indicates that the maximum reuse rate of waste is the reflection of the environmental protection benefits of the recycling network, and equation (10) indicates that the overall revenue of the entire recycling network system is maximized, which is a reflection of economic benefits

$$
\begin{gathered}
\sum_{j}^{\text {s.t. }} Z_{j k}^{Q W}=\sum_{k} Z_{k m}^{W E}+\sum_{k} Z_{k l}^{W R}+\sum_{k} Z_{k n}^{W T}, \quad \forall k, j, \\
\sum_{k} Z_{k m}^{W E}=Z_{m l}^{E T}+Z_{m n}^{E R}, \quad \forall k, \\
X_{k}^{W E} \cdot \sum_{j} Z_{j k}^{Q W}=\sum_{k} Z_{k m}^{W E}, \quad \forall k, \\
X_{k}^{W R} \cdot \sum_{j} Z_{j k}^{Q W}=\sum_{k} Z_{k l}^{W R}, \quad \forall k, \\
X_{k}^{W T} \cdot \sum_{j} Z_{j k}^{Q W}=\sum_{k} Z_{k n}^{W T}, \quad \forall k, \\
Z_{j k}^{Q W}=Y_{c}, \\
\sum_{j} Z_{j k}^{Q W} \leq \sum_{k} W_{k}, \quad \quad D \in(0,1) .
\end{gathered}
$$

Constraints (11) and (12) indicate the conservation of material quality at each logistics node, that is, the amount of industrial waste input at each waste generation point, artificial intelligence recycling classification center, remanufacturing center, resales center, and waste landfill center equal to the output amount; constraints (13) to (15) restrict the amount of waste transported by the classification center to each secondary node through a proportional limit; constraint (16) indicates that the predicted value in the artificial intelligence center is the waste input entered in the model; constraint (17) indicates the processing limit of each waste treatment center; and constraint (17) indicates the range of decision variables.

3.2.4. Multiobjective Function Solution Design. The above model belongs to a multiobjective mixed-integer programming model. Multiobjective programming generally belongs to the Pareto optimization problem. Because of the mathematical optimization problem involving multiple objective functions, it can only be as close as possible to the ideal solution based on the coordination of the objective functions. Based on the literature [23], this paper uses the fuzzy membership function in fuzzy theory to fuzzify the objective function and converts the multiobjective problem into a single-objective problem for solving. Specific steps are as follows:

Step 1: First, disassemble the dual objective function into two single-objective functions and use Lingo software to obtain the value interval of each objective function. First, the value range $\left[F_{1 \text { min }}, F_{1 \max }\right]$ of the first objective function is calculated by programming when only the 
TABLE 1: List of model parameters and descriptions.

\begin{tabular}{|c|c|c|c|}
\hline Node parameters & Description & Subscript & Operating cost \\
\hline$Q$ & Industrial waste generation point & : j & - \\
\hline W & AI recycling classification center & $: k$ & $\alpha$ \\
\hline$E$ & Remanufacturing center & $m$ & $\beta$ \\
\hline$R$ & Resale market & $l$ & $\lambda$ \\
\hline$T$ & Landfill center & $n$ & $\gamma$ \\
\hline Other parameters & \multicolumn{3}{|c|}{ Description } \\
\hline$\overline{W_{k}}$ & \multicolumn{3}{|c|}{ Maximum processing capacity per cycle of artificial intelligence recycling classification center } \\
\hline$Q_{j}(x, y)$ & \multicolumn{3}{|c|}{$j$ industrial waste generation point } \\
\hline$d$ & \multicolumn{3}{|c|}{ Distance between the two places (circle/km/ton) } \\
\hline$p_{k}^{w}$ & \multirow{2}{*}{\multicolumn{3}{|c|}{$\begin{array}{c}\text { Cost of constructing the } k \text { th artificial intelligence classification processing center } \\
\text { Reduced employee commissions for the construction of the } k \text { th artificial intelligence classification } \\
\text { processing center }\end{array}$}} \\
\hline$b_{k}^{w}$ & & & \\
\hline$Y_{c}$ & \multicolumn{3}{|c|}{ Artificial waste recycling center's forecasted garbage reception volume (tons) } \\
\hline$g$ & \multicolumn{3}{|c|}{ Average transportation cost (m/yuan) } \\
\hline General parameters & \multicolumn{3}{|c|}{ Description } \\
\hline$Z_{j k}^{Q W}$ & \multicolumn{3}{|c|}{$\begin{array}{l}\text { Amount of industrial waste transported from factories to artificial intelligence recycling sorting } \\
\text { centers over a period of time (tons) }\end{array}$} \\
\hline$Z_{k m}^{W E}$ & \multicolumn{3}{|c|}{$\begin{array}{l}\text { Amount of industrial waste transported from the intelligent sorting center to the remanufacturing } \\
\text { center over a period of time (tons) }\end{array}$} \\
\hline$Z_{k l}^{W R}$ & \multicolumn{3}{|c|}{$\begin{array}{c}\text { Amount of industrial waste transported from the intelligent sorting center to the resale market over } \\
\text { a period of time (tons) }\end{array}$} \\
\hline$Z_{m n}^{W T}$ & \multicolumn{3}{|c|}{$\begin{array}{c}\text { Amount of industrial waste transported from the intelligent sorting center to the landfill center } \\
\text { over a period of time }\end{array}$} \\
\hline$Z_{m l}^{E R}$ & \multirow{5}{*}{\multicolumn{3}{|c|}{$\begin{array}{l}\text { Amount of industrial waste from remanufacturing center to resale market over time (tons) } \\
\text { Amount of industrial waste from remanufacturing center to landfill center over time (tons) } \\
\text { Proportion of waste in AI recycling sorting centers shipped to remanufacturing centers } \\
\text { Proportion of waste shipped to resale markets in artificial intelligence recycling sorting centers } \\
\text { Proportion of wastes sent to landfill centers in artificial intelligence recycling sorting centers }\end{array}$}} \\
\hline$Z_{m n}^{m L}$ & & & \\
\hline$X_{\mathrm{k}}^{W E}$ & & & \\
\hline$X_{\mathrm{k}}^{W R R}$ & & & \\
\hline$X_{\mathrm{k}}^{W T}$ & & & \\
\hline Decision variables & \multicolumn{3}{|c|}{ Description } \\
\hline$D$ & \multicolumn{3}{|c|}{$\begin{array}{c}0-1 \text { variable, indicating whether to choose to set up a recycling point at } k \text {; if selected, take } 1 \text {, } \\
\text { otherwise take } 0\end{array}$} \\
\hline
\end{tabular}

environmental benefit target is considered; similarly, when only the economic benefits are considered, the value range of the second objective function can be obtained by programming calculation as $\left[F_{2 \text { min }}, F_{2 \max }\right]$.

Step 2: the membership degree fuzzy function method is used to assign a fuzzy wish value to each objective function, and the membership degree of the fuzzy wish is used to indicate the degree of satisfaction of the decisionmaker team in responding to the target level. This model belongs to the maximization optimization problem, and the membership function of its fuzzy desire is as follows:

$$
\begin{aligned}
& \mu_{1}= \begin{cases}1, & F_{1}>F_{1 \text { min }}, \\
\frac{F_{1}-F_{1 \text { min }}}{F_{1 \text { max }}-F_{1 \text { min }}}, & F_{1 \text { min }} \leq F_{1} \leq F_{1 \text { max }}, \\
0, & F_{1}<F_{1 \text { min }},\end{cases} \\
& \mu_{2}= \begin{cases}1, & F_{2}>F_{\text {min }}, \\
\frac{F_{2}-F_{2 \min }}{F_{2 \max }-F_{2 \min },} & F_{2 \min } \leq F_{2} \leq F_{2 \max }, \\
0, & F_{2}<F_{2 \min } .\end{cases}
\end{aligned}
$$

Step 3: Assign different weight coefficients to target satisfaction, unify the satisfaction of each target to form the overall satisfaction, so as to realize the conversion of multiple targets into a single target. That is, $\max F=\omega_{1} \mu_{1}+\omega_{2} \mu_{2}$, where $\omega_{1}$ and $\omega_{2}$ are the weight coefficients of the two membership fuzzy functions, which indicate the relative importance of the objective function and can be given by the industrial waste recycling network design planner based on the comprehensive analysis of the regional environmental and industrial policies.

Step 4: Use Lingo10 to solve the new objective function.

3.2.5. Analysis of Examples. A waste recycling company plans to deploy an artificial intelligence waste recycling and processing network in the industrial agglomeration area of City G. It is known that there are 20 existing waste-generating points in this area, a waste remanufacturing center, a waste resale center, and a waste landfill One for each center. The coordinates and related costs of each existing facility and those to be selected are shown in Table 2. Among them, Table 2 shows the coordinates of the existing remanufacturing center, sales center, and landfill center. Table 3 shows the coordinates and cost of the alternative artificial intelligence classification processing center, which is the maximum classification processing capacity in the period 
TABLE 2: Remanufacturing center, resale market, and landfill center.

\begin{tabular}{lcc}
\hline & $X$-axis & $Y$-axis \\
\hline Remanufacturing center & 35 & 85 \\
Resale market & 70 & 38 \\
Landfill center & 20 & 37 \\
\hline
\end{tabular}

TABLE 3: Coordinates of alternative points, construction cost, and cycle capacity.

\begin{tabular}{lcccc}
\hline Recycling classification center & $X$-axis & $Y$-axis & Construction cost (yuan) & Cycle capacity (tons) \\
\hline 1 & 76 & 12 & 200000 & 1200 \\
2 & 71 & 16 & 200000 & 1200 \\
3 & 68 & 25 & 200000 & 1200 \\
4 & 65 & 66 & 200000 & 1200 \\
5 & 55 & 47 & 200000 & 1200 \\
6 & 45 & 68 & 200000 & 1200 \\
7 & 35 & 41 & 200000 & 1200 \\
8 & 49 & 27 & 200000 & 1200 \\
\hline
\end{tabular}

TABLE 4: Coordinates of waste generation points, predicted value, and original classification costs.

\begin{tabular}{lccc}
\hline Waste generation point & $\begin{array}{c}X- \\
\text { axis }\end{array}$ & $\begin{array}{c}Y \text { - } \\
\text { axis }\end{array}$ & Cycle production (tons) \\
\hline 1 & 92 & 19 & 328 \\
2 & 22 & 47 & 235 \\
3 & 51 & 59 & 224 \\
4 & 89 & 92 & 166 \\
5 & 55 & 76 & 205 \\
6 & 14 & 76 & 362 \\
7 & 26 & 57 & 400 \\
8 & 84 & 7 & 280 \\
9 & 82 & 53 & 191 \\
10 & 93 & 94 & 161 \\
11 & 24 & 35 & 346 \\
12 & 57 & 45 & 263 \\
13 & 42 & 22 & 460 \\
14 & 1 & 79 & 135 \\
15 & 67 & 15 & 265 \\
16 & 98 & 48 & 158 \\
17 & 65 & 76 & 410 \\
18 & 37 & 85 & 260 \\
19 & 88 & 54 & 372 \\
20 & 29 & 67 & 340 \\
\hline
\end{tabular}

Table 4 shows the data use formulas (1)-(6) to predict the waste products in the next cycle of $G$ city. Table 5 shows the transportation ratio of each node. Table 6 shows the operating costs of processing waste per ton of waste at each node. In addition, according to the basic local conditions, the transportation cost is 4 yuan $/ \mathrm{ton} / \mathrm{km}$, the cost of traditional manual waste treatment is 200 yuan/ton, and the reuse rate of traditional industrial waste is $74.6 \%$.

Using Lingo software, the objective functions under different weights are solved, and the logistics network facilities location schemes under three weights and the corresponding objective function values of environmental and economic benefits of the network system are obtained (as shown in Table 7).
TABLE 5: Transportation proportion of each node after classification.

\begin{tabular}{lc}
\hline & Transportation ratio (\%) \\
\hline$X_{\mathrm{k}}^{W E}$ & 50 \\
$X_{\mathrm{k}}^{W R}$ & 30 \\
$X_{\mathrm{k}}^{W T}$ & 20 \\
\hline
\end{tabular}

TABLE 6: Operating costs of each node.

\begin{tabular}{lc}
\hline & Unit processing cost (yuan/ton/km) \\
\hline$Z_{j k}^{Q W}$ & 4 \\
$Z_{k m}^{W E}$ & 3 \\
$Z_{k l}^{W R}$ & 3 \\
$Z_{k n}^{W T}$ & 3 \\
$Z_{m l}^{E R}$ & 3 \\
$Z_{m n}^{E T}$ & 3 \\
\hline
\end{tabular}

It can be known from the example that when only environmental protection benefits are used as the objective function, alternative points $1,2,4,5,6,7$, and 8 need to be selected intelligence to build an artificial classification center. The economic benefits generated at this time are 174,206 yuan per unit cycle. The environmental protection benefit is to achieve $84.6 \%$ of the reuse rate of waste; when only economic benefits are considered and when only economic benefits are used as the objective function, alternative points $2,3,4,5,6$, and 8 need to be selected. During the construction of artificial intelligence classification center, the economic benefit generated at this time is 288,014 yuan per cycle, and the environmental protection benefit is to achieve $82.7 \%$ of the reuse rate of waste; when considering both economic and environmental benefits, at this time, alternative points $2,4,5,6,7$, and 8 are selected. At this time, the economic benefit is 246,137 yuan per cycle, and the environmental protection benefit is to achieve $83.5 \%$ of the reuse rate of the waste. This plan takes into account both economic and environmental benefits. Therefore, the solution of the model in this paper is shown in Figure 6 at this time. 
TABle 7: Logistics network facility location plan.

\begin{tabular}{lccc}
\hline $\begin{array}{l}\text { Weight } \\
\text { coefficient } \\
\left(\omega_{1}, \omega_{2}\right)\end{array}$ & $\begin{array}{c}\text { Total } \\
\text { network cost } \\
\text { (yuan) }\end{array}$ & $\begin{array}{c}\text { Landfill } \\
\text { rate }(\%)\end{array}$ & $\begin{array}{c}\text { AI recycling } \\
\text { classification center } \\
\text { alternative point }\end{array}$ \\
\hline$(1,0)$ & 174206 & 84.6 & $1,2,4,5,6,7,8$ \\
$(0,1)$ & 288014 & 82.7 & $2,3,4,5,6,8$ \\
$(1,1)$ & 246137 & 83.5 & $2.4,5,6,7,8$ \\
\hline
\end{tabular}

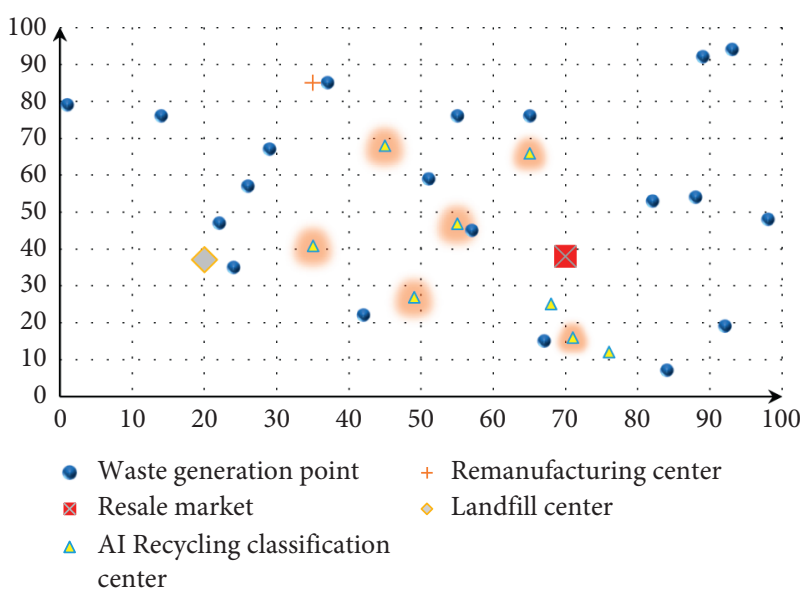

FIgURE 6: Distribution of site selection schemes.

\section{Conclusion and Discussion}

With the acceleration of China's industrialization and urbanization process, the traditional urban waste treatment and logistics network has been unable to meet the rapidly expanding population and production capacity, and the large accumulation of waste in industrial parks has become a major cause of environmental pollution. One of the main challenges of the sustainable development strategy is to solve the problem of upgrading and designing waste network systems in industrial parks.

Based on artificial intelligence's prediction technology and image recognition technology, this article intelligently upgrades the traditional industrial waste planning and management system, designs an industrial waste intelligent classification center with intelligent prediction and intelligent classification capabilities, and implements its key implementation. The technology was explored. Finally, the value and significance of the intelligent classification center designed in this paper are illustrated by constructing a multiobjective site selection model considering both economic and environmental benefits.

It can be seen from the different weighted results of economic and environmental benefits of fuzzy mathematical methods that regardless of focusing on economic benefits or environmental benefits, huge economic benefits can be obtained by reducing the dependence on human resources after building a new intelligent classification center. Therefore, relevant departments and responding companies can be advised to fully apply such artificial intelligence technology to the existing industrial waste recycling network, thereby enhancing the global benefits of the existing industrial waste recycling network, improving system efficiency and robustness, and ultimately achieve green sustainable development.

In previous research, [11] proposed an urban waste recycling system based on radio frequency wireless networks. Using sensor technology and radio frequency wireless network technology, the information collection of urban waste bins and the apex and positioning of urban trash cans were achieved. This has a certain enlightening effect on us. Big data technology and artificial intelligence technology are gradually changing our way of life. Therefore, this article uses new artificial intelligence technology to optimize the existing industrial waste recycling network and realize the idea of intelligent, economical, and sustainable recycling systems. It can promote academic innovation and disciplinary intersection in the field of waste management. In [16], while considering the cost, and taking into account the benefits of investment in environmental protection, a green supply chain network design model was proposed, and the optimization method was used to study the supply network construction cost and the minimum environmental pollution of the entire supply chain system. Their research found that the main way to reduce carbon dioxide emissions and total costs is to increase network capacity and enhance the service capabilities of facilities, which coincides with our research conclusions. To some extent, this shows that the only way for the supply chain network to achieve greening and maximize economic benefits is to improve service capabilities and response capabilities through technological improvements. References $[17,19]$, etc., proposed or applied classic multiobjective function tools such as particle swarm optimization and genetic algorithms when solving multiobjective function problems. The multiobjective function algorithm designed in this paper uses the fuzzy membership function in the fuzzy theory to obfuscate the objective function and converts the multiobjective problem into a single-objective problem for solving. On the one hand, the solution process and results can be more clearly explained and analyzed. For example, the effect of the objective function on the overall system optimization under different preferences can be further analyzed. On the other hand, only 0 and 1 were the two extreme weights. Researchers can also design new solving algorithms based on different weight preferences.

Our further research direction is to consider more factors in the supply chain, such as transportation mode, demand uncertainty, artificial intelligence learning ability, image recognition fault tolerance rate, etc., in order to enhance its applicability to real-world scenarios. On the contrary, we can also extend our research by designing new solutions to solve this multiobjective model.

\section{Data Availability}

The data used to support the findings of this study are available from the corresponding author upon request.

\section{Conflicts of Interest}

The authors declare that there are no conflicts of interest regarding the publication of this paper. 


\section{Acknowledgments}

This work was supported by the Construction Project for Domestic 2017 First-Class-Discipline in Guizhou Province (GNYL[2017]005), Guizhou Provincial Philosophy and Social Science Planning Joint Fund (18GZLH03), and Major project fund for social science \& humanities of Guizhou University (GDZT201702).

\section{References}

[1] Z. Lu, J. Zhang, S. Lu et al., "Pollution characteristics and evaluation of heavy metal elements in the soil around the municipal solid waste incineration power plant and in the plant area," Environmental Science, vol. 40, no. 5, pp. 4832492, 2019.

[2] Y. Peng, L. Lei, X. Peng, P. Yang, X. Zhao, and W. Ma, “The development of domestic waste classification in China? Obstacles and countermeasures," China Environmental Science, vol. 38, no. 10, pp. 3874-3879, 2018.

[3] L. Dan, G. Chen, W. Ma, and N. Duan, "Characteristics and treatment status of domestic garbage in villages and towns in China," China Environmental Science, vol. 38, no. 11, pp. 4187-4197, 2018.

[4] T. Lindhqvist, Extended Producer in Cleaner Production: Policy Principle to Promote Environmental Improvements of Product Systems, IIIEE, Lund, Sweden, 2000.

[5] J. Li, D. Li, and M. Lu, "Research on the design of C2B waste mobile phone reverse logistics recycling network based on carbon emission," Manufacturing Automation, vol. 40, no. 9, pp. 95-101, 2018.

[6] Y. Chen, Y. Yang, and Q. Zhang, "Design of reverse logistics network for waste household appliances based on third party recycling," Mathematics in Practice and Theory, vol. 46, no. 17, pp. 81-89, 2016.

[7] D. Wang, H. Du, and W. Yang, "A two-stage fourth-party reverse logistics network design model based on CVaR," Industrial Engineering and Management, vol. 20, no. 1, pp. 22-27, 2015.

[8] H. Prajapati, R. Kant, and R. Shankar, "Bequeath life to death: state-of-art review on reverse logistics," Journal of Cleaner Production, vol. 211, pp. 503-520, 2019.

[9] S. Agrawal, R. K. Singh, and Q. Murtaza, "A literature review and perspectives in reverse logistics," Resources, Conservation and Recycling, vol. 97, pp. 76-92, 2015.

[10] D. Huang and Y. Li, "Problems in urban domestic waste treatment in Wuhan and suggestions for improvement," Resources and Industries, vol. 14, no. 3, pp. 167-173, 2012.

[11] L. I. Yan, G. Yang, and A. Ren, "Research on urban garbage recycling system based on radio frequency wireless network," Modern Electronic Technology, vol. 38, no. 16, pp. 48-50, 2015.

[12] W. Wang, X. Ge, and Y. Xu, "Analysis of multi-agent interest game and differential responsibility in urban waste separation and recycling," China Population, Resources and Environment, vol. 27, no. S2, pp. 41-44, 2017.

[13] Q. Wang, Li Xia, and C. Tong, "Selection and evaluation of reverse logistics suppliers based on program satisfaction," Logistics Technology, vol. 37, no. 11, pp. 50-55, 2018.

[14] Q. Zhang and X. Lu, "Research on e-commerce return vehicle routing problem and ant colony algorithm," Computer Engineering and Applications, vol. 54, no. 22, pp. 239-245, 2018.

[15] M. Lu, Z. Ye, J. Tian, and F. Wang, "Optimization design of reverse logistics network considering random reproducibility ratio," System Engineering, vol. 35, no. 6, pp. 113-120, 2017.
[16] F. Wang, X. Lai, and N. Shi, “A multi-objective optimization for green supply chain network design," Decision Support Systems, vol. 51, no. 2, pp. 262-269, 2011.

[17] S. Elhedhli and M. Ryan, "Green supply chain network design to reduce carbon emissions," Transportation Research Part D: Transport and Environment, vol. 17, no. 5, pp. 370-379, 2012.

[18] Li Rui, M. Huang, and F. Sun, "Multi-to-multiple network design of fourth-party logistics with hard time windows," Control Engineering, vol. 25, no. 10, pp. 1927-1933, 2018.

[19] Q. Zhang and W. Li-rong, "Multi-objective stochastic programming model for reverse logistics network design," Soft Science, vol. 29, no. 10, pp. 120-124, 2015.

[20] S. Yang and L. Huang, "Financial early warning model of listed companies based on BP neural network," Systems Engineering Theory \& Practice, vol. 25, no. 1, pp. 12-18, 2005.

[21] W. Yang, H. Wang, J. Zhang et al., "An improved vehicle realtime detection algorithm based on Faster-RCNN," Journal of Nanjing University (Natural Science Edition), vol. 55, no. 2, 2019.

[22] K. Simonyan and A. Zisserman, "Very deep convolutional networks for large-scale image recognition," 2014, https:// arxiv.org/abs/1409.1556.

[23] X. Xu and J. Li, Theory and Method of Multi-Objective Decision Making, Tsinghua University Press, Beijing, China, 2005. 